OPEN ACCESS

Edited by:

Rinaldo Wellerson Pereira

Catholic University of Brasilia

(UCB), Brazi

Reviewed by:

Longbiao Guo,

Chinese Academy of Agricultural

Sciences, China

Guilherme Borba Neumann,

Humboldt University of

Berlin, Germany

*Correspondence:

Jian-Feng Liu

liujf@cau.edu.cn

†These authors have contributed equally to this work

Specialty section:

This article was submitted to Evolutionary and Population Genetics,

a section of the journal

Frontiers in Genetics

Received: 10 April 2020

Accepted: 15 January 2021

Published: 04 February 2021

Citation:

Du H, Zheng X, Zhao Q, Hu Z, Wang $H$, Zhou L and Liu J-F (2021) Analysis of Structural Variants Reveal

Novel Selective Regions in the Genome of Meishan Pigs by Whole

Genome Sequencing.

Front. Genet. 12:550676.

doi: $10.3389 /$ fgene.2021.550676

\section{Analysis of Structural Variants Reveal Novel Selective Regions in the Genome of Meishan Pigs by Whole Genome Sequencing}

\author{
Heng Du ${ }^{1+}$, Xianrui Zheng ${ }^{1+}$, Qiqi Zhao ${ }^{1}$, Zhengzheng Hu ${ }^{1}$, Haifei Wang ${ }^{2}$, Lei Zhou ${ }^{1}$ and \\ Jian-Feng Liu ${ }^{1 *}$ \\ ${ }^{1}$ National Engineering Laboratory for Animal Breeding, Key Laboratory of Animal Genetics, Breeding and Reproduction, \\ Ministry of Agriculture, College of Animal Science and Technology, China Agricultural University, Beijing, China, ${ }^{2}$ College of \\ Animal Science and Technology, Yangzhou University, Yangzhou, China
}

Structural variants (SVs) represent essential forms of genetic variation, and they are associated with various phenotypic traits in a wide range of important livestock species. However, the distribution of SVs in the pig genome has not been fully characterized, and the function of SVs in the economic traits of pig has rarely been studied, especially for most domestic pig breeds. Meishan pig is one of the most famous Chinese domestic pig breeds, with excellent reproductive performance. Here, to explore the genome characters of Meishan pig, we construct an SV map of porcine using whole-genome sequencing data and report 33,698 SVs in 305 individuals of 55 globally distributed pig breeds. We perform selective signature analysis using these SVs, and a number of candidate variants are successfully identified. Especially for the Meishan pig, 64 novel significant selection regions are detected in its genome. A 140-bp deletion in the Indoleamine 2,3-Dioxygenase 2 (IDO2) gene, is shown to be associated with reproduction traits in Meishan pig. In addition, we detect two duplications only existing in Meishan pig. Moreover, the two duplications are separately located in cytochrome P450 family 2 subfamily $\mathrm{J}$ member 2 (CYP2J2) gene and phospholipase A2 group IVA (PLA2G4A) gene, which are related to the reproduction trait. Our study provides new insights into the role of selection in SVs' evolution and how SVs contribute to phenotypic variation in pigs.

Keywords: genome structural variants, whole-genome sequencing, selection signature, reproduction trait, pig

\section{INTRODUCTION}

Structural variants (SVs) are significant contributors to genetic diversity, and it includes insertions/deletions (InDels), inversions, translocations and copy number variations (CNVs) (Alkan et al., 2011; Tattini et al., 2015). SVs can be canonically classified as two types: "balanced" SVs and "unbalanced" SVs (Escaramís et al., 2015; Collins et al., 2017). "Balanced" SVs refer to rearrangements hardly causing major gain or loss of genomic DNA, including inversions and translocations. "Unbalanced" SVs, containing deletions, insertions of novel sequences and duplications, which can result in differences in the numbers of base pairs (bp) among individual genomes (Escaramís et al., 2015; Collins et al., 2017). Genetic variations can contribute to variations 
in phenotypic traits; previous studies have indicated that SVs were more significantly related to phenotypic diversity than single nucleotide polymorphisms (SNPs) (Chiang et al., 2017). However, SNPs and CNVs are shown to capture 83.6 and $17.7 \%$ of total genetic variation in gene expression, respectively (Stranger et al., 2007). Thus, the study of SVs can improve our understanding of the evolution of populations, phenotypic polymorphisms, and the functional genome.

SVs are widespread among different individuals and cell types, such as in non-inherited somatic (Zhuang and Weng, 2015) and germline cells (Guan and Sung, 2016). SVs (>50 bp) can influence large segments of genomes and produce genomic dosage effects (Zhou et al., 2011; Lupski, 2015; Rice and McLysaght, 2017). SV maps of the human, mouse, dog, and many other species have already been successfully constructed (Yalcin et al., 2011, 2012; Berglund et al., 2012; Radke and Lee, 2015; Sudmant et al., 2015). Many genes with SV mutations have also been found to be associated with economic traits in domestic animals. For example, a 110-Kb deletion in the MIMT1 gene was associated with abortion and stillbirth in cattle (Flisikowski et al., 2010). Similarly, a 176-Kb duplication containing the PTLR gene and SPEF2 gene influenced the growth of chicken feathers (Elferink et al., 2008). The white coat of boars was caused by a 450-Kb duplication involving the KIT gene (Giuffra et al., 2002).

SVs have been able to be more precisely detected in pigs with the fast development of genome sequencing. For example, Paudel et al. detected that CNVs might play a role in olfaction, and CNVs were correlated with porcine fatty acid composition and growth traits (Paudel et al., 2015; Revilla et al., 2017). Meanwhile, SVs were discovered in Chinese domestic pigs using whole-genome sequencing (WGS) (Yang et al., 2017). In a previous study, we detected SV regions from a sample size of 13 pigs from Asia and Europe, and found a hotspot region in chromosome X of Asian pig breeds (Zhao et al., 2016). However, for the Meishan pig, as one of the most important Chinese domestic breeds with excellent reproduction features, there is no detailed study of SVs and their functions for this breed.

Here, to investigate the breed-specific SVs in the Meishan pig, we analyzed the SV landscape across the pig genome using WGS data generated from Meishan pig and other 54 pig breeds. Especially, 42 samples (almost 1/8 of all samples) were Meishan pigs to ensure the accuracy of detected SVs in Meishan pigs. We employed a combined detection strategy to detect various types of SVs by running six different software. In this study, we obtained an integrated SV map of 33,698 variants for 305 individuals. Simultaneously, among these variants, 20,770 SVs were detected in the Meishan pig genome. In addition, we identified three critical functional genes correlated with reproduction traits in Meishan pigs. This study provides novel insights into SVs in the pig genome and their potential roles in selective and evolutionary processes in pigs.

\section{MATERIALS AND METHODS WGS Data Processing}

To detect SVs in the pig genome, we downloaded WGS data of 305 pigs from NCBI (https://www.ncbi.nlm.nih.gov/) and
EBI (http://www.ebi.ac.uk/). These pigs included 55 breeds from Africa, Europe, Asia, South-east Asia, and Central America, and they were further divided into domesticated boars and wild boars (Figure 1A). Detailed information on these pigs listed in Supplementry Table 1 . Raw reads were trimmed to a minimum base quality of 20 using NGSQC Toolkit v2.3.3 (Patel and Jain, 2012). Simultaneously, NGSQC Toolkit was also used to remove adapters, low-quality reads (mean quality scores lower than 20 ), and the reads which were shorter than $70 \mathrm{bp}$ after trimming. The filtered reads were aligned to the Sus scrofa 11.1 reference genome using Burrows-Wheeler Aligner v 0.7.17 (Li and Durbin, 2009). Raw mapping results were reordered and sorted by SAMtools v 1.9 (Li et al., 2009) and Picard v1.119. Genome Analysis Toolkit (GATK) v 3.8 (DePristo et al., 2011) was used to correct each individual's alignment results with marking duplicates, local realigning around indels and base quality score recalibration procedures. The read-depth statistics of all downloaded samples were calculated using the DepthOfCoverge function in GATK.

\section{Whole-Genome Detection of SVs}

To enhance SVs' detection accuracy and sensitivity, we used six software involving three computational algorithms (read-pair, read-depth, and split-read methods). These software included Breakdancer v 1.1 (Chen et al., 2009), Pindel v 0.2.5b8 (Ye et al., 2009), DELLY v0.8.1 (Rausch et al., 2012), Manta v 1.5.0 (Chen et al., 2016), Genomestrip v 2.00.1940 (Handsaker et al., 2015), and CNVnator v0.4 (Abyzov et al., 2011).

To control the frequency of false positives and archive a unified pig SV map, we merged the results from all software based on the following consensus principles:

(a) Merged loci (except for translocations) should show $>75 \%$ reciprocal overlap among different software;

(b) The site of SVs was retained when two or more software simultaneously detected the site;

(c) The start and end positions of SVs were determined using the median method. This median method involved selecting the sites that corresponded to the same SV firstly, and then the median of the start and end positions of the selected sites was calculated as the merged SV's start and end positions.

Lastly, SVs used in downstream analyses were further filtered by rules of deletions ( $>50 \mathrm{bp},<10 \mathrm{Mb}$ ), insertions ( $>50 \mathrm{bp}$, $<10 \mathrm{Mb})$, inversions $(<10 \mathrm{Mb})$, duplications $(<10 \mathrm{Mb})$, and translocations (Supplementary Notes).

\section{Identification of SV Breakpoints}

We used a local assembly method to identify SV breakpoints and assess the validity of detected SVs. First, the sequences of candidate SVs in our call set were assembled by falling back to TIGRA-SV v 0.3.7 (Chen et al., 2014). For each site, we used TIGRA-SV to extract the reads and generate local assembly sequences. The sequences around each SV of the reference genome were then used as the reference sequences. Finally, AGE software v0.4 (Abyzov and Gerstein, 2011) was applied to align the assembled sequences of each locus to the corresponding reference and to select validated sites. 


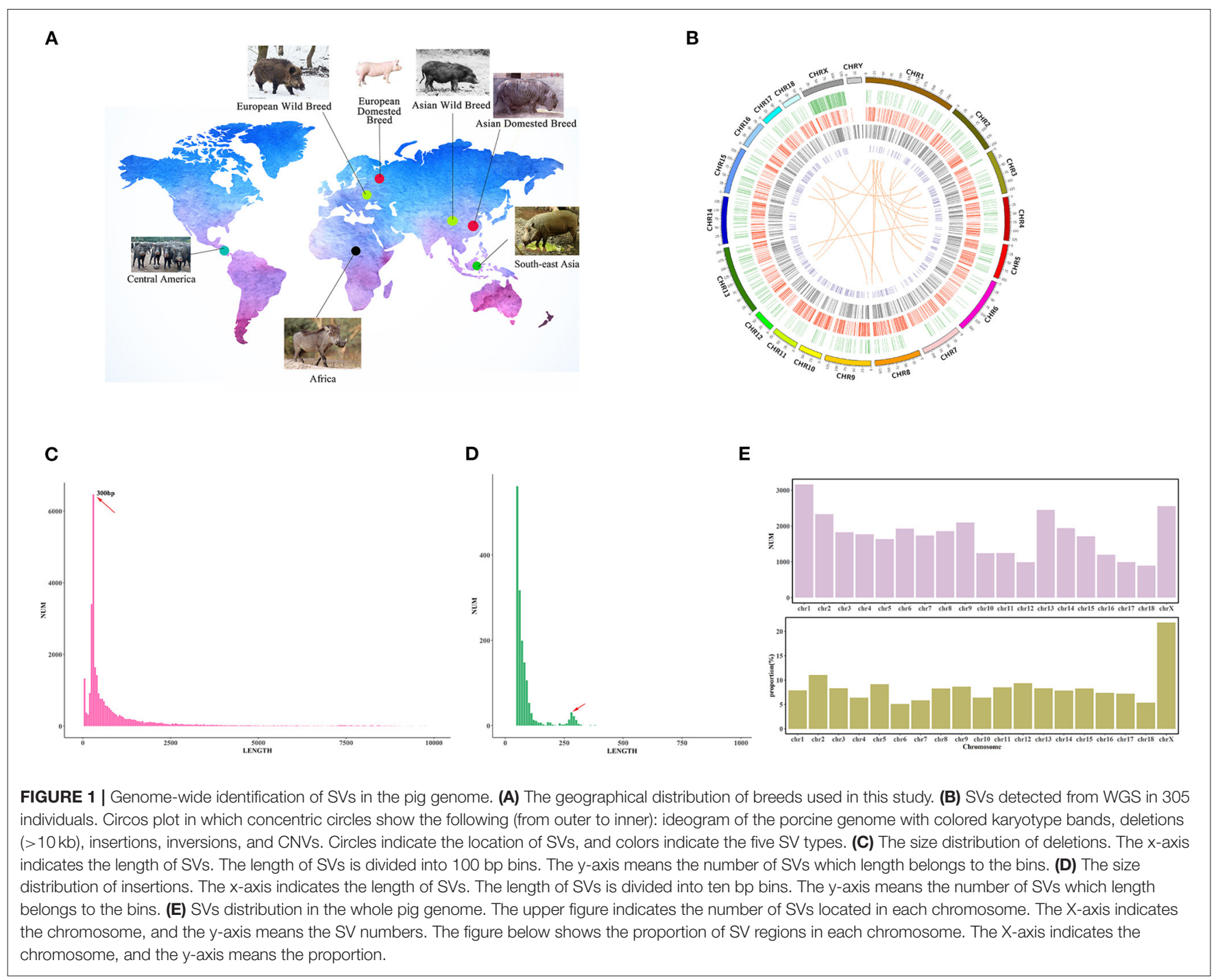

\section{SNP Calling}

GATK and SAMtools were used to call SNPs. The Haplotypecaller pipeline of GATK was used to detect and genotype variants. The results of SAMtools were used to select reliable SNP sites from the GATK outputs. The "HardFiltration" function of GATK was then implemented to refine the merged SNP call set (Supplementary Notes).

\section{Population Genetic Structure and Admixture}

To infer the population structure of pigs using detected SVs, we first extracted the genotype predicted by three software (Delly, Manta and Genomestrip), which contained the genotyping process among all six software. The genotype inferred simultaneously by at least two software, was considered as the genotype of a site. All SVs in the SV call set were then genotyped using SVTyper v0.1.4 (Chiang et al., 2015). Next, deletions, SNPs, and SNPs combined with deletions were separately used to estimate the relationships among all populations in our study. Then, we used these data to perform principal component analyses (PCA) (Yang et al., 2011). MEGA-X (Kumar et al., 2018) was employed to construct the phylogenetic tree based on identity-by-Descent (IBD) matrices of the three datasets, respectively. The IBD matrices were calculated by PLINK v1.7(Purcell et al., 2007) using the parameters "-cluster -distance-matrix." The population structure analysis was conducted by Admixture v1.3 (Alexander et al., 2009). We also estimated the genetic background for each population. Nine possible groupings $(\mathrm{K}=2$ to $\mathrm{K}=10$ ) were calculated by Admixture, and the results were plotted using our R scripts.

\section{Selection Signature Analysis}

Deletions were selected as genetic markers to characterize potential selective signatures in Meishan pigs. In this discovery pipeline, deletions over $100 \mathrm{~Kb}$ in length were removed. The absolute allele frequency difference $\triangle \mathrm{AF}$ between the Meishan population and the other two populations (Duroc and Tibetan wild boars) $\left(\triangle \mathrm{AF}=\right.$ abs $\left[\mathrm{AltAF}_{\text {Meishan }}-\right.$ mean $\left(\mathrm{AltAF}_{\text {Duroc }}\right.$ 
+ AltAF $\left.\mathrm{F}_{\text {Tibetan }}\right)$ ]) was calculated in home-written Perl scripts, and it was used to detect selective sweeps in Meishan pigs. Sites in the top $5 \%$ of the $\triangle \mathrm{AF}$ distribution were considered to show signatures of selection. We also used the relative frequency difference (RFD) (Zhou et al., 2015) to detect regions showing selective signatures. The RFD was applied to measure differentiation in the deletions of populations based on variation frequency. The following formula calculated RFD:

$$
R F D=\frac{F_{\text {Meishan }}-\text { mean }\left(F_{\text {Duroc }}+F_{\text {Tibetan }}\right)}{F_{\text {population }}}
$$

where $F_{\text {Meishan }}, F_{\text {Duroc }}, F_{\text {Tibetan }}$, and $F_{\text {population }}$ represent the frequencies of deletions in Meishan, Duroc, Tibetan, and the three populations (all samples), respectively. Deletions with low frequencies $(<0.01)$ were removed. Sites in the top $5 \%$ of the RFD distribution were considered to be under significant selection. Sites that were claimed as significant both by $\triangle \mathrm{AF}$ and RFD were added to the selective signature datasets.

\section{Gene Annotation and Enrichment Analysis}

Gene-based and region-based annotations were conducted for all detected SVs in ANNOVAR v 2019Oct24 (Wang et al., 2010). There were 63,041 transcripts (including 13,184 transcripts without coding sequence annotations) for 31,907 unique genes within the Duroc genome used to annotate SVs, and the genes were downloaded from the Ensemble database (S. scrofa 11.1). We performed gene enrichment analysis for the annotated genes with KOBAS v3.0 (Mao et al., 2005). We used Fisher's exact test and hypergeometric test as the significance test method. The Benjamini-Hochberg and Benjamini-Yekutieli methods were applied to correct the false discovery rate. Pathways with corrected $P$-values smaller than 0.05 were considered to be significant.

\section{Identification of Deletions in Genes}

Reads aligned to regions with deletions were extracted from each sample's BAM files by bedtools v2.28.0 (https://bedtools. readthedocs.io/). SOAPdenovo2-r241 (Luo et al., 2012) was implemented in assembling the contigs, and the contigs were aligned to the reference sequence by blastn (version 2.9.0+) (ftp://ftp.ncbi.nlm.nih.gov/blast/executables/blast+/LATEST/).

The splicing sites were predicted by the Alternative Splice Site Predictor (Wang and Marin, 2006).

\section{Validation of the SVs by Polymerase Chain Reaction (PCR)}

We designed primers using Primer5 (http://www.premierbiosoft. com/primerdesign) for SVs validation (Supplementary Table 2). The DNA templates combined with primers were processed under preheating $\left(93^{\circ} \mathrm{C}, 5 \mathrm{~min}\right)$, amplification (denaturation at $93^{\circ} \mathrm{C}, 40 \mathrm{~s}$, then annealing at $68^{\circ} \mathrm{C}, 30 \mathrm{~s}$, finally elongation at $72^{\circ} \mathrm{C}, 60 \mathrm{~s}$, these process executed 33 cycles $)$, and extension $\left(72^{\circ} \mathrm{C}\right.$, $7 \mathrm{~min}$ ) processes. The PCR products from Meishan pig, Tibetan wild boar, and Duroc were separated by size in a $1 \%$ agarose gel electrophoresis. SVs were considered successfully validated if their PCR products successfully matched to expected sizes and locations.

\section{RESULTS}

\section{SV Map Construction of the Pig Genome}

We collected WGS data (100-bp read lengths) from 305 pigs of 55 breeds (Li et al., 2013; Ai et al., 2015; Frantz et al., 2015; Zhao et al., 2018) (Supplementary Table 1). After removing low-quality reads, a total of $10,663 \mathrm{~Gb}$ of sequencing data with an average $10 \times$ mapped read depth was aligned to S. scrofa 11.1. We merged six software results to construct an integrated SV map, and the six software contained three detection algorithms: read-pair, read-depth, and split-read algorithms. The high-quality genotyped SVs that we obtained consisted of 30,749 indels (29,173 deletions and 1,576 insertions), 393 inversions, 19 translocations, and 2,537 CNVs (Figure 1B). The comparison of the detected SVs and the public SV database of Ensemble (release 101), showed that 18.43\% of SVs identified in our study were new (Supplementary Figure 1). Furthermore, to validate the reliability of the SV map, we applied a local assembly algorithm. We obtained an average alignment rate of $94.08 \%$ (Supplementary Figure 2), indicating that our SV map was of high quality. Meanwhile, we randomly validated eight SVs with PCR amplification, and they existed in Meishan pig and Tibetan wild boars, respectively. These eight SVs were successfully confirmed and yielded robust PCR products with the expected sizes in these two breeds, respectively (Supplementary Table 2 ). In addition, among all the SVs detected in Meishan pig, there were 18,690 indels (17,690 deletions and 1,000 insertions), 112 inversions, 8 translocations, and 1,960 CNVs.

Additionally, we found that the length of all SVs amounted to $212 \mathrm{Mb}$, which was $8.4 \%$ of the total length of the pig genome. Meanwhile, the SV map revealed that CNV occupied the largest proportion of the genome (5.2\% of whole genome) among all SV variants, and insertions contributed to the smallest proportion of the genome $(0.005 \%$ of the genome) (Supplementary Figure 3). These observations demonstrate that the accumulation of repetitive sequences occurred relatively frequently during the pig genome's evolution (Frantz et al., 2016). Interestingly, we found that SVs were not equally occurred in different chromosomes. The proportions of SVs (total length of SVs in the chromosome/total length of the chromosome) in chromosome X (21.84\%) and chromosome 2 $(11.87 \%)$ were higher than other chromosomes (no more than $10 \%$ ) (Figure 1E). This phenomenon might be correlated to the various haplotype regions in chromosome $\mathrm{X}$ of different pig breeds.

The average size for deletions, insertions and inversions were $2,032 \mathrm{bp}, 87 \mathrm{bp}$, and $62,419 \mathrm{bp}$, respectively. For CNVs, the average size was 50,345 bp. We divided all of the identified SVs into four different length groups $(50 \mathrm{bp}-1 \mathrm{~Kb}, 1 \mathrm{~Kb}-10 \mathrm{~Kb}$, $10 \mathrm{~Kb}-100 \mathrm{~Kb}$, and $100 \mathrm{~Kb}-1 \mathrm{Mb})$. We found that deletions and insertions were enriched in the $50 \mathrm{bp}-1 \mathrm{~Kb}$ group, while inversions and $\mathrm{CNV}$ s were enriched in the $10 \mathrm{~Kb}-100 \mathrm{~Kb}$ group (Supplementary Figure 4). Interestingly, further exploration of the lengths of deletions and insertions in the 50 bp to $1 \mathrm{~Kb}$ group revealed that the size of these deletions and insertions tended to be $\sim 300 \mathrm{bp}$ in length (Figures 1C,D). A previous study 
suggested that the enrichment of indels of the tRNAGlu-derived short interspersed element (SINE/tRNAGlu) contributed to the distribution of indels of this length (Li et al., 2017).

\section{Annotation of Pig SVs}

We used ANNOVAR to annotate all high-confidence SV calls against features in the S. scrofa 11.1 annotation. Many SVs were located in intergenic and intronic regions, with 45.81 and $6.56 \%$ within $1 \mathrm{~kb}$ of a protein-coding gene or long non-coding RNA gene, respectively. $44.37 \%$ of all SVs overlapped one or more Ensemble genes. Meanwhile, we also detected SNPs of 305 pigs and compared the annotation results of these SNPs to SVs. Unexpectedly, we found that the distribution of SVs in the pig genome was similar to SNPs' distribution. However, in contrast to SNPs, we found that SVs occurred more frequently in exonic (3.18 vs. $0.78 \%$ ) and lncRNA exonic regions (1.92 vs. $1.18 \%$ ) (Supplementary Figures 5, 6). This difference indicates that SVs might be more likely to play a role in altering patterns of gene expression than SNPs.

\section{Population Genetic Structure Analysis}

We separately used SVs, SNPs, and SNPs combined with SVs (SNPs+SVs) to infer the population genetic structure of all 55 breeds. Because of their more comprehensive genotyping results, deletions were employed as the exclusive representative of SVs in this analysis. Previous studies have demonstrated the effectiveness of using biallelic deletions in population genetic analyses (Sudmant et al., 2015; Bertolotti et al., 2020; Guo et al., 2020). PCA of "SNPs+SVs," SVs and SNPs all indicated that European populations were clearly distinct from Asian boar populations. A novel finding was that we detected a clear separation between African Phacochoerus africanus populations and South-east Asian Sus populations ( $S$. barbatus, S. cebifrons, S. celebensis, and $S$. verrucosus) by SV-based PCA; however, this separation was not recovered by "SNPs+SVs"-based PCA and SNP-based PCA (Figure 2A). Meanwhile, the above three PCA analyses consistently indicated that Meishan pig clustered with other Asian domestic pigs.

We constructed phylogenetic trees for all populations (Tibetan wild boars were divided into nine subpopulations according to different sampling locations) based on the above "SNPs+SVs," SVs or SNPs data, respectively. P. africanus was used as the outgroup. All populations were divided into four major branches: four breeds from South-east Asia, Sumatran pig, Asian lineages, and European lineages (Figure 2B). Meishan pig was significantly attributed to Asian lineages, and it had close genetic relationships with other Asian domestic breeds.

We then further performed a structure analysis for all the 55 breeds, and the number of presumed ancestral populations $(\mathrm{K})$ was set from 2 to 10 . We observed similar patterns when $\mathrm{K}$ ranged from 2 to 5 with three different datasets (Supplementary Figures 7-9). In summary, our final set of deletion genotypes captured the expected population genetic structure.

\section{Analysis of Selective Signals in Meishan Pigs}

Svs were known to influence the genome significantly, and they were often associated with specific traits (Sudmant et al., 2015). Our study explored whether the SVs were selected during breed formation and their relationships with specific agricultural traits.

To test whether SVs experienced positive selection during the breed formation of specific breeds, we further analyzed three breeds, including Meishan, Duroc, and Tibetan wild boars. These three breeds each had their unique traits, like the high reproduction of Meishan pig, cold and low-oxygen tolerant of Tibetan wild boars, and high lean yield of Duroc pig. In this study, we only focused on one trait-high reproduction. We used two different methods to detect SVs under significant selective sweeps. We first used the $\triangle \mathrm{AF}$ statistic to identify selective signature regions, followed by an RFD test. Only sites discovered by both $\triangle \mathrm{AF}$ and RFD were considered to be selective signature sites (Figures 3A,B, and Supplementary Figure 10). Finally, a total of 64 deletion regions were found to be under selection (Supplementary Table 3), which covered 37 proteincoding genes. The gene enrichment analysis revealed that these genes were enriched in 16 Gene Ontology pathways (corrected $P<0.05$, Supplementary Table 4). Among these 37 functionally annotated genes, we identified an important functional gene, IDO2, in which a 140-bp deletion occurred in the intronic region only for Meishan pigs (Figures 4A-D).

Furthermore, we predicted the splice sites within the deletion region. This region contained an alternative $5^{\prime}$ splice site and a constitutive $3^{\prime}$ splice site. After removing this deletion, this region appeared two alternative $5^{\prime}$ splice sites and four alternative $3^{\prime}$ splice sites (Supplementary Figure 11 and Supplementary Table 5). Thus, this finding indicated that the alternative splicing and expression level of IDO2 might be affected by this deletion. IDO2 played a vital role in maintaining the pregnancy of vertebrate animals (Clark et al., 2005). Simultaneously, dysfunction in IDO2 could lead to recurrent spontaneous abortion, preeclampsia, preterm labor, and fetal growth restriction (Chang et al., 2018).

\section{The Role of Other Non-deletion SVs in Meishan Pigs}

To identify potential roles in other types of SVs except for deletions, we selected 170 duplications and 77 insertions that specifically existed in Meishan pigs and not in Duroc and Tibetan wild boar populations (Supplementary Tables 6, 7). We annotated these sites to the pig genome to focus on genes that were influenced by these sites.

We detected 84 functional annotated genes, which overlapped with the 170 duplications. Among these genes, we found two genes, cytochrome P450 family 2 subfamily $\mathrm{J}$ member 2 (CYP2J2) and phospholipase A2 group IVA (PLA2G4A), were especially responsible for the ovarian steroidogenesis pathway. This pathway is critical for normal uterine function, the establishment and maintenance of pregnancy, and mammary gland development. Notably, a duplication of $115 \mathrm{~kb}$ was observed in the CYP2J2 only for Meishan pigs 
(Supplementary Figure 12). Besides, a duplication of length 4,668 bp was detected in PLA2G4A, which was only existed in Meishan pig (Supplementary Figure 12). Previous studies have reported that PLA2G4A was often up-regulated and contributed to ovarian hormone synthesis (Diouf et al., 2006) and the estrus cycle (Ababneh and Troedsson, 2013). PLA2G4A might thus be a potential candidate gene for fertility traits. Therefore, the expression of these two genes was likely affected by the duplicate sequences of these two genes.

\section{DISCUSSION}

Here, we detected SVs across the genome of pigs using six software with NGS data from 55 pig breeds. The SV map identified 6,209 new SVs and greatly contributed to the public SV dataset. To study the relationship of SVs and the specific traits of Meishan pig, we performed selection signature analysis with Meishan pig, Duroc pig and Tibetan wild boar using deletions. Besides, we also discovered the other particular SVs which were only existed in Meishan pig and further predicted their functions. Finally, we identified critical functional genes associated with SVs related to the reproduction traits of pigs. This research provides new insights into the role of selection in the evolution of SVs and candidate genes for porcine fertility traits.

With the population genetic structure analysis results using "SNP+SVs," SVs and SNPs, we observed that SVs could capture the expected genetic structure. This finding indicated that SVs, especially for deletions, had the power to discriminate divergences among distantly related lineages. Therefore, SVs might also be considered in future studies of genetic structure analysis. In recent years, SV markers have become a promising and useful tool for forensic identification (Caputo et al., 2017) and biogeographic research (Levy-Sakin et al., 2019). Previous studies have successfully used SVs to characterize the genetic diversities and genetic relationships in many species, like human

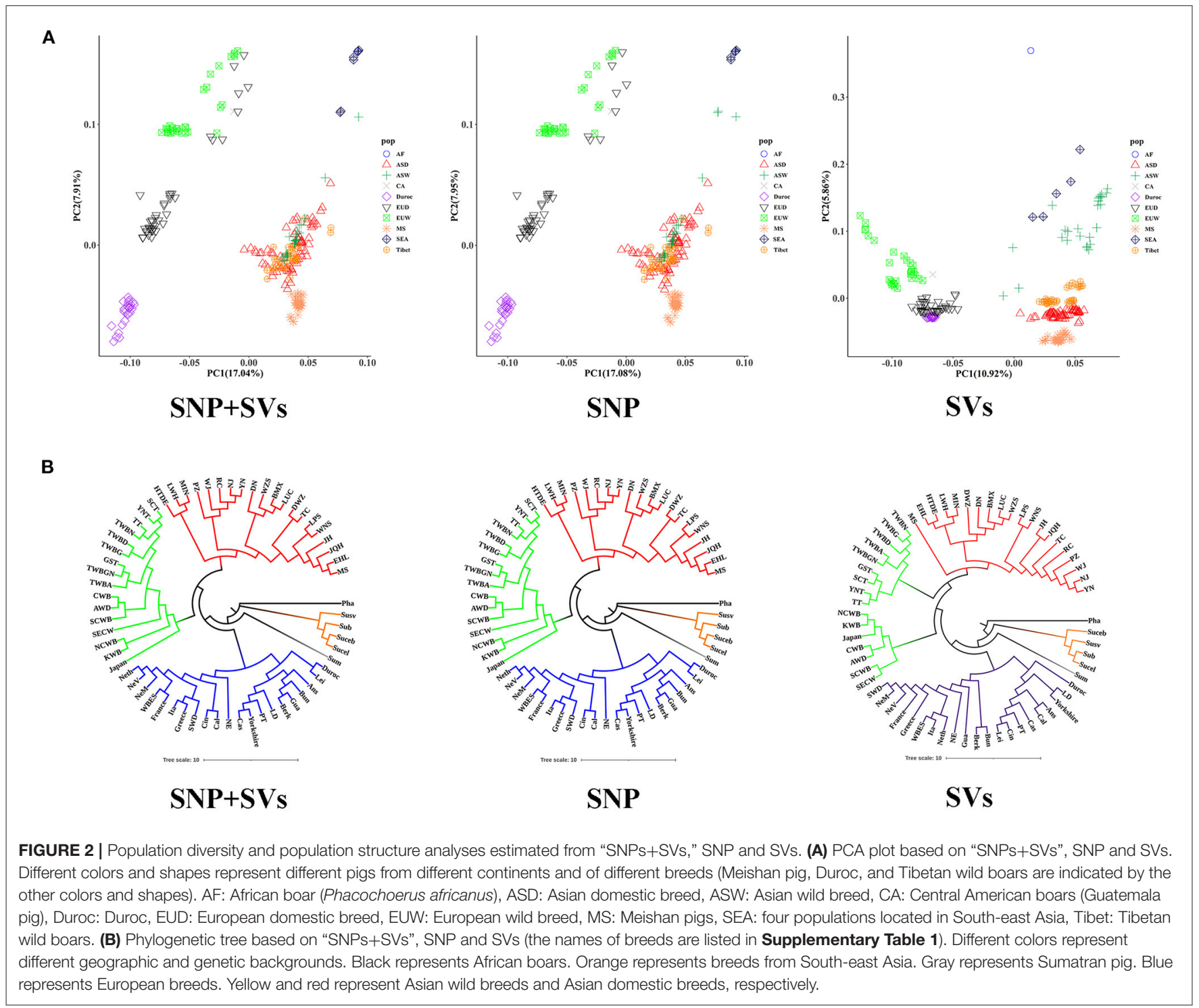


A



B

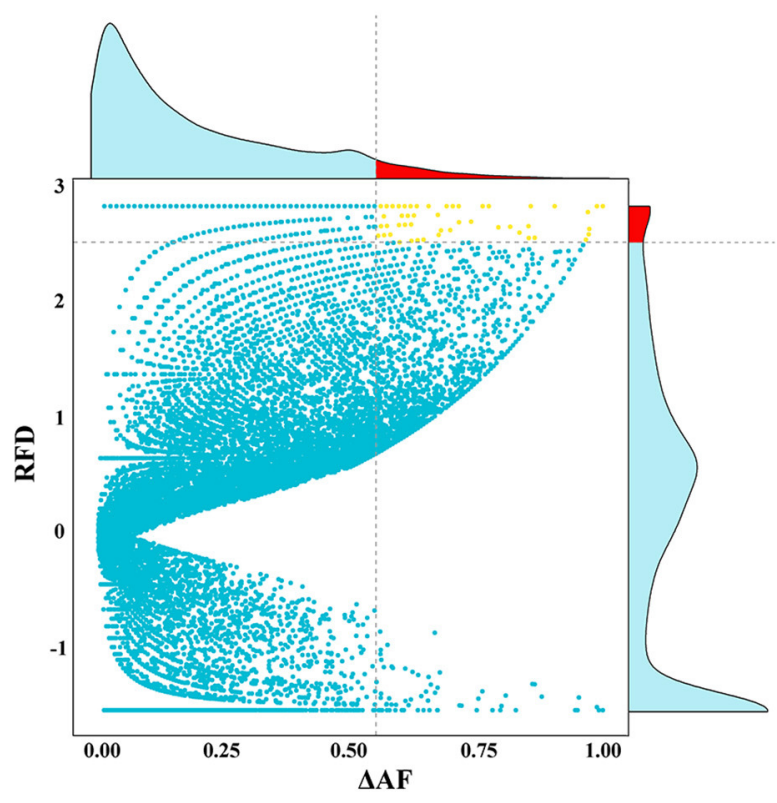

FIGURE 3 | Selective signal analyses using deletion data. (A) The plot of RFD values among Meishan, Duroc, and Tibetan wild boar populations. The genome-wide distribution of RFD is calculated using deletion data. The X-axis represents the chromosome. Y-axis indicates the RFD value. (B) Definition of selective sweep regions for the Meishan population using deletions. The $x$-axis corresponds to the $\triangle \mathrm{AF}$ value, and the $\mathrm{y}$-axis corresponds to the RFD value. The horizontal dashed line and vertical dashed line indicated the top $5 \%$ of RFD values and the top $5 \%$ of $\triangle \mathrm{AF}$ values, respectively. The upper-frequency figure means the frequency of $\Delta \mathrm{AF}$. The red region indicates the top $5 \%$ of $\triangle \mathrm{AF}$ values. The right frequency figure represents the frequency of RFD. The red region indicated the top $5 \%$ of $\Delta \mathrm{AF}$ values.

groups (Xie et al., 2018), peach accessions (Guo et al., 2020), and salmon groups (Bertolotti et al., 2020). These findings also demonstrated the high power of SVs in population analyses. Thus, our results provided a preliminary framework for how to apply SVs in genetic structure analysis.

Our study discovered that $44.37 \%$ of all SVs overlapped one or more Ensemble genes, slightly higher than SNPs (43.07\%). Additionally, because of long base pairs for each SV, exonic regions were covered more frequently by SVs than SNPs. This indicated that SVs might have more tight relationships with traits. A previous study showed that candidate variants associated with peach fruit traits were identified in genome-wide association studies (GWAS) using SVs (Guo et al., 2020). Meanwhile, Song et al. demonstrated that presence and absence variationsGWAS (PAV-GWAS) complement SNP-GWAS in identifying associations to traits (Song et al., 2020). SVs deserve further research in variants association analysis for different traits.

One of the consequences of SVs being targets of selection was differences in SV frequencies among populations (Zhou et al., 2015). Thus, analysis of variation in the frequencies of SVs among different populations might promote the identification of important genomic regions under selection and functional genes in these regions. Compared with our previous study examining selective signals in Meishan pigs using SNPs (Zhao et al., 2018), we obtained 64 non-overlapping selective signature regions and identified a potential functional gene, $I D O 2$, associated with pig fertility. Previous studies have shown that IDO2 played a vital role in the maintenance of pregnancy in vertebrate animals (Moraes et al., 2018). The intronic variation could alter the expression of proteins by influencing the alternative splicing of relevant genes (Xu et al., 2018). We found a loss of a small segment in IDO2, which affected its alternative splicing. We speculated that this loss might influence the expression level of IDO2 and affected fertility traits in Meishan pigs. DNA methylation was linked to the regulation of $I D O 2$ expression and altered patterns of $I D O 2$ expression; however, DNA methylation can also adversely affect the outcomes of pregnancy (Spinelli et al., 2019). Thus, IDO2 could be further targeted for the future epigenomic study of porcine fertility. 
A

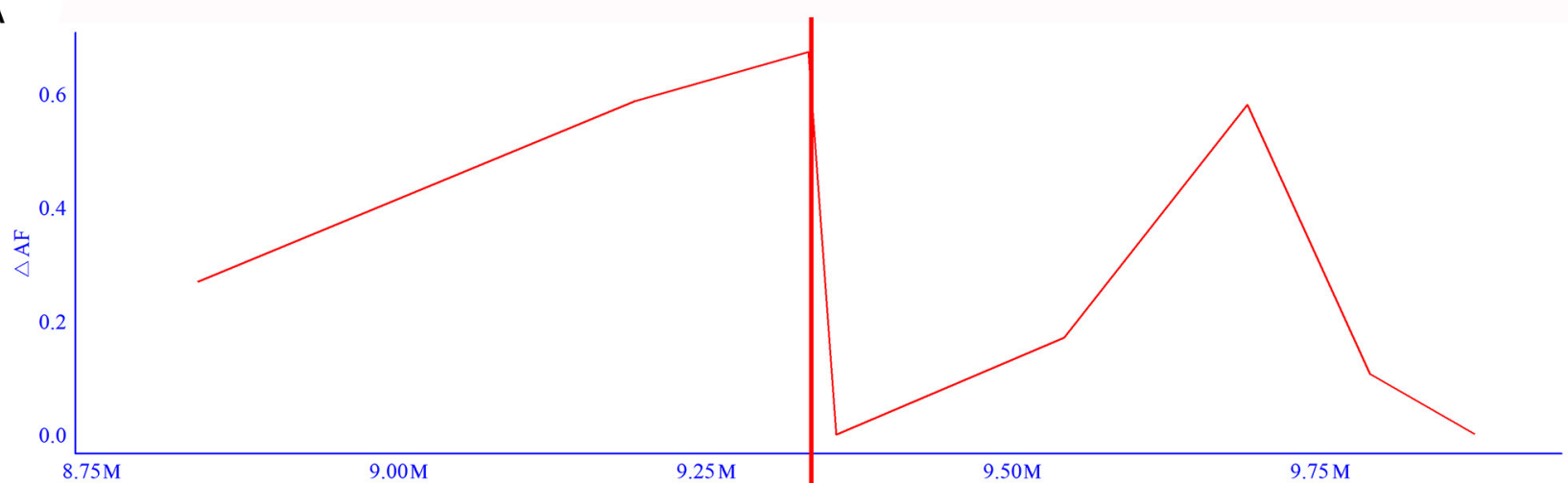

B

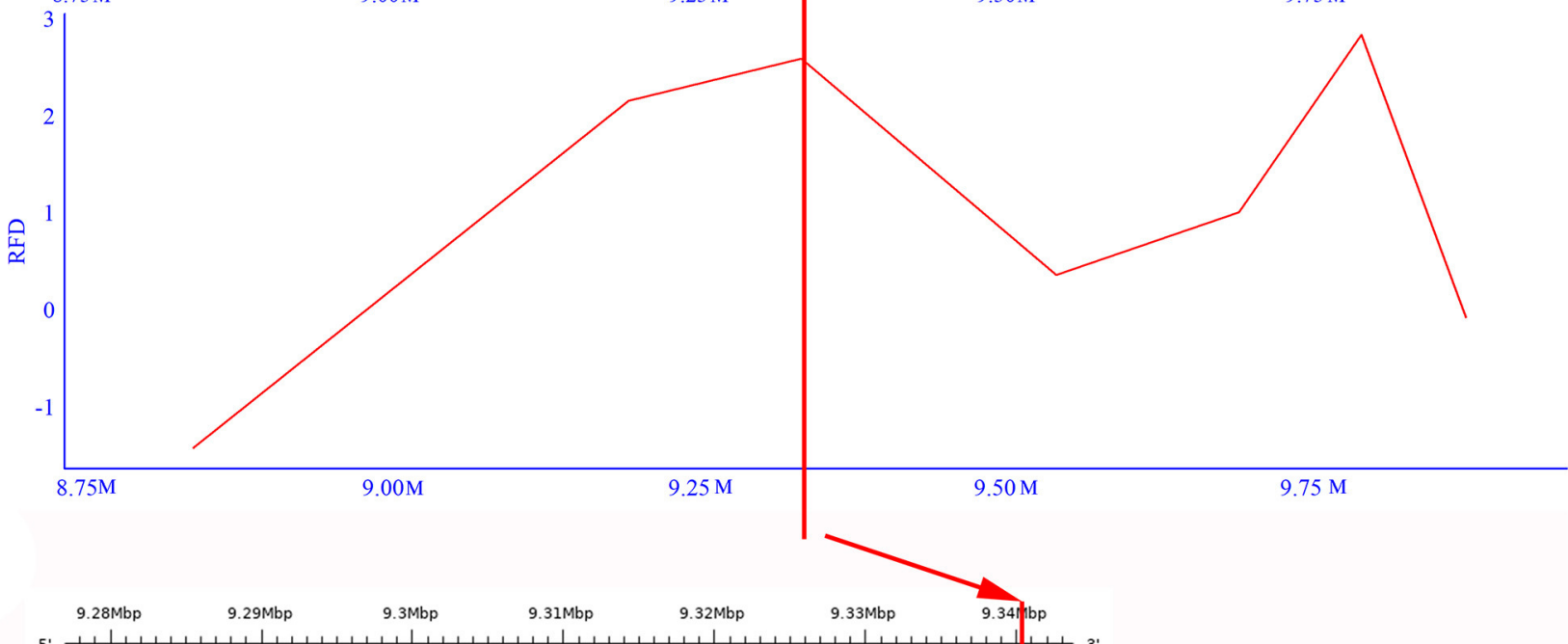

C

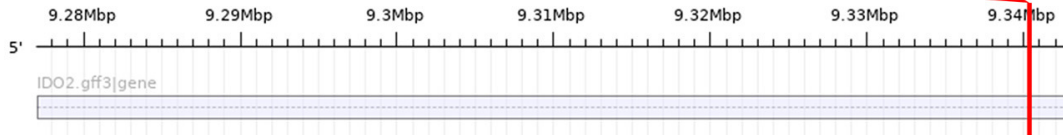

DO2 gff 3 m mRNA

ENSSSCT00000030402

ENSSCT00000056091

D

Reference ATGTCTACAAGCCAAAGAGCCAGCAA

CAGAAGGAGCTCAGGGTG

Duroc

ATGTCTACAAGCCAAAGAGCCAGCAA

CAGAAGGAGCTCAGGGTG

Tibetan

ATGTCTACAAGCCAAAGAGCCAGCAA CAGAAGGAGCTCAGGGTG

Meishan

ATGTCTACAAGCCAAAGAGCCAGCAAAG.

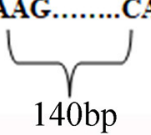

CAGAAGGAGCTCAGGGTG

$140 \mathrm{bp}$

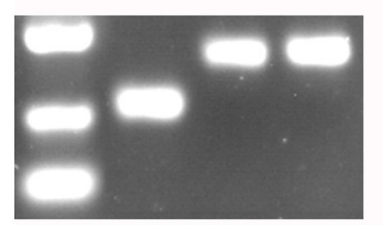

Marker Meishan Tibetan Duroc

FIGURE 4 | Detected deletion in IDO2 gene. (A) The $\triangle$ AF values of deletions around IDO2 gene. X-axis means the region around the IDO2 gene in chromosome 17. Y-axis indicates $\triangle \mathrm{AF}$ value. (B) The RFD values of deletions around IDO2 gene. Y-axis indicates the RFD value. (C) The gene structure of IDO2 gene. The horizontal blue bar represents the gene region, and the horizontal gray bar indicates transcript. (D) Deletions in IDO2 gene of Meishan pig. The left chart indicates the sequence of different breeds in the deleted region. The right chart shows PCR results to detect this deletion in Meishan, Tibetan wild boar, and Duroc breeds.

We also investigated important functional genes strongly correlated with other traits of Meishan pigs covered by the SVs except for deletions. For instance, we found that a duplication influenced the PLA2G4A gene. Previous studies have shown that this gene was associated with premature ovarian failure (POF), which could cause amenorrhoea, infertility, and early onset of menostasis (Kuang et al., 2014). In the pig industry, POF syndrome often reduces the productive lifespan of sows and 
can cause substantial economic losses. Thus, PLA2G4A could be targeted in future studies of POF in both pigs and humans. Overall, our findings contributed to further understanding and exploring the genome characteristics of Meishan pig and other pig breeds.

\section{DATA AVAILABILITY STATEMENT}

The datasets presented in this study can be found in online repositories. The names of the repository/repositories and accession number(s) can be found in the article/Supplementary Material.

\section{AUTHOR CONTRIBUTIONS}

J-FL conceived and designed the experiments. HD performed all analysis processes and wrote the manuscript. XZ and QZ

\section{REFERENCES}

Ababneh, M. M., and Troedsson, M. H. T. (2013). Ovarian steroid regulation of endometrial phospholipase A2 isoforms in horses. Reprod. Dom. Anim. 48, 311-316. doi: 10.1111/j.1439-0531.2012.02151.x

Abyzov, A., and Gerstein, M. (2011). AGE: defining breakpoints of genomic structural variants at single-nucleotide resolution, through optimal alignments with gap excision. Bioinformatics 27, 595-603. doi: 10.1093/bioinformatics/btq713

Abyzov, A., Urban, A. E., Snyder, M., and Gerstein, M. (2011). CNVnator: an approach to discover, genotype, and characterize typical and atypical CNVs from family and population genome sequencing. Gen. Res. 21, 974-984. doi: 10.1101/gr.114876.110

Ai, H. S., Fang, X. D., Yang, B., Huang, Z. Y., Chen, H., Mao, L. K., et al. (2015). Adaptation and possible ancient interspecies introgression in pigs identified by whole-genome sequencing. Nat. Genet. 47:217. doi: 10.1038/ng.3199

Alexander, D. H., Novembre, J., and Lange, K. (2009). Fast model-based estimation of ancestry in unrelated individuals. Gen. Res. 19, 1655-1664. doi: 10.1101/gr.094052.109

Alkan, C., Coe, B. P., and Eichler, E. E. (2011). Genome structural variation discovery and genotyping. Nat. Rev. Genet. 12, 363-376. doi: 10.1038/nrg2958

Berglund, J., Nevalainen, E. M., Molin, A. M., Perloski, M., Andre, C., Zody, M. C., et al. (2012). Novel origins of copy number variation in the dog genome. Gen. Biol. 13:R73. doi: 10.1186/gb-2012-13-8-r73

Bertolotti, A. C., Layer, R. M., Gundappa, M. K., Gallagher, M. D., Pehlivanoglu, E., Nome, T., et al. (2020). The structural variation landscape in 492 Atlantic salmon genomes. Nat. Commun. 11, 5176. doi: 10.1038/s41467-020-18972-x

Caputo, M., Amador, M. A., Santos, S., and Corach, D. (2017). Potential forensic use of a $33 \mathrm{X}$-InDel panel in the Argentinean population. Int. J. Legal Med. 131, 107-112. doi: 10.1007/s00414-016-1399-Z

Chang, R. Q., Li, D. J., and Li, M. Q. (2018). The role of indoleamine-2,3dioxygenase in normal and pathological pregnancies. Am. J. Rep. Immunol. 79:e12786. doi: 10.1111/aji.12786

Chen, K., Chen, L., Fan, X., Wallis, J., Ding, L., and Weinstock, G. (2014). TIGRA: a targeted iterative graph routing assembler for breakpoint assembly. Gen. Res. 24, 310-317. doi: 10.1101/gr.162883.113

Chen, K., Wallis, J. W., McLellan, M. D., Larson, D. E., Kalicki, J. M., Pohl, C. S., et al. (2009). BreakDancer: an algorithm for high-resolution mapping of genomic structural variation. Nat. Methods 6, 677-U676. doi: $10.1038 /$ nmeth. 1363

Chen, X. Y., Schulz-Trieglaff, O., Shaw, R., Barnes, B., Schlesinger, F., Kallberg, M., et al. (2016). Manta: rapid detection of structural variants and indels for germline and cancer sequencing applications. Bioinform. 32, 1220-1222. doi: 10.1093/bioinformatics/btv710 performed PCR validation of structural variation. J-FL, ZH, HW, and LZ revised the paper. All authors read and approved the final manuscript.

\section{FUNDING}

This work was financially supported by the National Natural Science Foundations of China (31661143013, 31972563), Beijing Natural Science Foundation (6192010), and the Beijing Municipal Commission of Science and Technology (Z191100004019009).

\section{SUPPLEMENTARY MATERIAL}

The Supplementary Material for this article can be found online at: https://www.frontiersin.org/articles/10.3389/fgene. 2021.550676/full\#supplementary-material

Chiang, C., Layer, R. M., Faust, G. G., Lindberg, M. R., Rose, D. B., Garrison, E. P. et al. (2015). SpeedSeq: ultra-fast personal genome analysis and interpretation. Nature Methods 12, 966-968. doi: 10.1038/nmeth.3505

Chiang, C., Scott, A. J., Davis, J. R., Tsang, E. K., Li, X., Kim, Y., et al. (2017). The impact of structural variation on human gene expression. Nat. Genet. 49:692. doi: 10.1038/ng.3834

Clark, D. A., Blois, S., Kandil, J., Handjiski, B., Manuel, J., and Arck, P. C. (2005). Reduced uterine indoleamine 2,3-dioxygenase vs. increased Th1/Th2 cytokine ratios as a basis for occult and clinical pregnancy failure in mice and humans. Am. J. Reprod. Immunol. 54, 203-216. doi: 10.1111/j.1600-0897.2005. 00299.x

Collins, R. L., Brand, H., Redin, C. E., Hanscom, C., Antolik, C., Stone, M. R., et al. (2017). Defining the diverse spectrum of inversions, complex structural variation, and chromothripsis in the morbid human genome. Genome Biol. 18:36. doi: 10.1186/s13059-017-1158-6

DePristo, M. A., Banks, E., Poplin, R., Garimella, K. V., Maguire, J. R., Hartl, C., et al. (2011). A framework for variation discovery and genotyping using next-generation DNA sequencing data. Nat. Genet. 43:491. doi: 10.1038/ng.806

Diouf, M. N., Sayasith, K., Lefebvre, R., Silversides, D. W., Sirois, J., and Lussier, J. G. (2006). Expression of phospholipase A2 group IVA (PLA2G4A) is upregulated by human chorionic gonadotropin in bovine granulosa cells of ovulatory follicles. Biol. Reprod. 74, 1096-1103. doi: 10.1095/biolreprod.105.048579

Elferink, M. G., Vallee, A. A. A., Jungerius, A. P., Crooijmans, R. P. M. A., and Groenen, M. A. M. (2008). Partial duplication of the PRLR and SPEF2 genes at the late feathering locus in chicken. BMC Genom. 9:391. doi: 10.1186/1471-2164-9-391

Escaramís, G., Docampo, E., and Rabionet, R. (2015). A decade of structural variants: description, history and methods to detect structural variation. Brief. Funct. Gen. 14, 305-314. doi: 10.1093/bfgp/elv014

Flisikowski, K., Venhoranta, H., Nowacka-Woszuk, J., McKay, S. D., Flyckt, A., Taponen, J., et al. (2010). A novel mutation in the maternally imprinted PEG3 domain results in a loss of MIMT1 expression and causes abortions and stillbirths in cattle (Bos taurus). PLOS ONE 5:e15116. doi: 10.1371/journal.pone.0015116

Frantz, L., Meijaard, E., Gongora, J., Haile, J., Groenen, M. A. M., and Larson, G. (2016). The evolution of suidae. Ann. Rev. Anim. Biosci. 4, 61-85. doi: 10.1146/annurev-animal-021815-111155

Frantz, L. A. F., Schraiber, J. G., Madsen, O., Megens, H. J., Cagan, A., Bosse, M., et al. (2015). Evidence of long-term gene flow and selection during domestication from analyses of Eurasian wild and domestic pig genomes. Nat Genet. 47:1141. doi: 10.1038/ng.3394

Giuffra, E., Tornsten, A., Marklund, S., Bongcam-Rudloff, E., Chardon, P., Kijas, J. M. H., et al. (2002). A large duplication associated with dominant white color in 
pigs originated by homologous recombination between LINE elements flanking KIT. Mamm Genome 13, 569-577. doi: 10.1007/s00335-002-2184-5

Guan, P., and Sung, W.-K. (2016). Structural variation detection using next-generation sequencing data. Methods 102, 36-49. doi: 10.1016/j.ymeth.2016.01.020

Guo, J., Cao, K., Deng, C., Li, Y., Zhu, G., Fang, W., et al. (2020). An integrated peach genome structural variation map uncovers genes associated with fruit traits. Genome Biol 21:258. doi: 10.1186/s13059-020-02169-y

Handsaker, R. E., Van Doren, V., Berman, J. R., Genovese, G., Kashin, S., Boettger, L. M., et al. (2015). Large multiallelic copy number variations in humans. Nat. Genet. 47:296. doi: 10.1038/ng.3200

Kuang, H. X., Han, D. W., Xe, J. M., Yan, Y. X., Li, J., and Ge, P. L. (2014). Profiling of differentially expressed microRNAs in premature ovarian failure in an animal model. Gynecol. Endocrinol. 30, 57-61. doi: 10.3109/09513590.2013.850659

Kumar, S., Stecher, G., Li, M., Knyaz, C., and Tamura, K. (2018). MEGA X: molecular evolutionary genetics analysis across computing platforms. Mol. Biol. Evol. 35, 1547-1549. doi: 10.1093/molbev/msy096

Levy-Sakin, M., Pastor, S., Mostovoy, Y., Li, L., Leung, A. K. Y., McCaffrey, J., et al. (2019). Genome maps across 26 human populations reveal population-specific patterns of structural variation. Nat. Commun. 10:7. doi: 10.1038/s41467-019-08992-7

Li, H., and Durbin, R. (2009). Fast and accurate short read alignment with Burrows-Wheeler transform. Bioinformatics 25, 1754-1760. doi: 10.1093/bioinformatics/btp324

Li, H., Handsaker, B., Wysoker, A., Fennell, T., Ruan, J., Homer, N., et al. (2009). The sequence alignment/map format and SAMtools. Bioinformatics 25, 2078-2079. doi: 10.1093/bioinformatics/btp352

Li, M. Z., Chen, L., Tian, S. L., Lin, Y., Tang, Q. Z., Zhou, X. M., et al. (2017). Comprehensive variation discovery and recovery of missing sequence in the pig genome using multiple de novo assemblies. Gen. Res. 27, 865-874. doi: 10.1101/gr.207456.116

Li, M. Z., Tian, S. L., Jin, L., Zhou, G. Y., Li, Y., Zhang, Y., et al. (2013). Genomic analyses identify distinct patterns of selection in domesticated pigs and Tibetan wild boars. Nat. Genet. 45, 1431-U1180. doi: 10.1038/ng.2811

Luo, R. B., Liu, B. H., Xie, Y. L., Li, Z. Y., Huang, W. H., Yuan, J. Y., et al. (2012). SOAPdenovo2: an empirically improved memory-efficient short-read de novo assembler. Gigascience 1:18. doi: 10.1186/2047-217X-1-18

Lupski, J. R. (2015). Structural variation mutagenesis of the human genome: Impact on disease and evolution. Environ. Mol. Mutag. 56, 419-436. doi: 10.1002/em.21943

Mao, X., Cai, T., Olyarchuk, J. G., and Wei, L. (2005). Automated genome annotation and pathway identification using the KEGG Orthology (KO) as a controlled vocabulary. Bioinformatics 21, 3787-3793. doi: 10.1093/bioinformatics/bti430

Moraes, J. G. N., Behura, S. K., Geary, T. W., Hansen, P. J., Neibergs, H. L., and Spencer, T. E. (2018). Uterine influences on conceptus development in fertility-classified animals. Proc. Natl. Acad. Sci. U.S.A. 115, E1749-E1758. doi: $10.1073 /$ pnas. 1721191115

Patel, R. K., and Jain, M. (2012). NGS QC toolkit: a toolkit for quality control of next generation sequencing data. PLoS ONE 7:e30619. doi: 10.1371/journal.pone.0030619

Paudel, Y., Madsen, O., Megens, H. J., Frantz, L. A., Bosse, M., Crooijmans, R. P., et al. (2015). Copy number variation in the speciation of pigs: a possible prominent role for olfactory receptors. BMC Gen. 16:330. doi: 10.1186/s12864-015-1449-9

Purcell, S., Neale, B., Todd-Brown, K., Thomas, L., Ferreira, M. A. R., Bender, D., et al. (2007). PLINK: A tool set for whole-genome association and population-based linkage analyses. Am. J. Hum. Gen. 81, 559-575. doi: 10.1086/ 519795

Radke, D. W., and Lee, C. (2015). Adaptive potential of genomic structural variation in human and mammalian evolution. Brief. Funct. Genom. 14, 358-368. doi: 10.1093/bfgp/elv019

Rausch, T., Zichner, T., Schlattl, A., Stutz, A. M., Benes, V., and Korbel, J. O. (2012). DELLY: structural variant discovery by integrated paired-end and split-read analysis. Bioinform. 28, i333-i339. doi: 10.1093/bioinformatics/ bts 378
Revilla, M., Puig-Oliveras, A., Castello, A., Crespo-Piazuelo, D., Paludo, E., Fernandez, A. I., et al. (2017). A global analysis of CNVs in swine using whole genome sequence data and association analysis with fatty acid composition and growth traits. PLOS ONE 12:e0177014. doi: 10.1371/journal.pone.0177014

Rice, A. M., and McLysaght, A. (2017). Dosage sensitivity is a major determinant of human copy number variant pathogenicity. Nat. Commun. 8:14366. doi: $10.1038 /$ ncomms 14366

Song, J. M., Guan, Z., Hu, J., Guo, C., Yang, Z., Wang, S., et al. (2020). Eight high-quality genomes reveal pan-genome architecture and ecotype differentiation of Brassica napus. Nat. Plants 6, 34-45. doi: 10.1038/s41477-0190577-7

Spinelli, P., Latchney, S. E., Reed, J. M., Fields, A., Baier, B. S., Lu, X., et al. (2019). Identification of the novel Idol imprinted locus and its potential epigenetic role in pregnancy loss. Hum Mol. Genet. 28, 662-674. doi: 10.1093/hmg/ ddy 383

Stranger, B. E., Forrest, M. S., Dunning, M., Ingle, C. E., Beazley, C., Thorne, N., et al. (2007). Relative impact of nucleotide and copy number variation on gene expression phenotypes. Science 315, 848-853. doi: 10.1126/science.1136678

Sudmant, P. H., Rausch, T., Gardner, E. J., Handsaker, R. E., Abyzov, A., Huddleston, J., et al. (2015). An integrated map of structural variation in 2,504 human genomes. Nature 526:75. doi: 10.1038/nature15394

Tattini, L., D’Aurizio, R., and Magi, A. (2015). Detection of genomic structural variants from next-generation sequencing data. Front. Bioengin. Biotechnol. 3:92. doi: 10.3389/fbioe.2015.00092

Wang, K., Li, M. Y., and Hakonarson, H. (2010). ANNOVAR: functional annotation of genetic variants from high-throughput sequencing data. Nucleic Acids Res. 38:e164. doi: 10.1093/nar/gkq603

Wang, M., and Marin, A. (2006). Characterization and prediction of alternative splice sites. Gene 366, 219-227. doi: 10.1016/j.gene.2005.07.015

Xie, T., Guo, Y. X., Chen, L., Fang, Y. T., Tai, Y. C., Zhou, Y. S., et al. (2018). A set of autosomal multiple InDel markers for forensic application and population genetic analysis in the Chinese Xinjiang Hui group. Forensic Sci Int Gen. 35, 1-8. doi: 10.1016/j.fsigen.2018.03.007

Xu, W., He, H., Zheng, L., Xu, J. W., Lei, C. Z., Zhang, G. M., et al. (2018). Detection of 19-bp deletion within PLAG1 gene and its effect on growth traits in cattle. Gene 675, 144-149. doi: 10.1016/j.gene.2018.06.041

Yalcin, B., Wong, K., Agam, A., Goodson, M., Keane, T. M., Gan, X. C., et al. (2011). Sequence-based characterization of structural variation in the mouse genome. Nature 477, 326-329. doi: 10.1038/nature10432

Yalcin, B., Wong, K., Bhomra, A., Goodson, M., Keane, T. M., Adams, D. J., et al. (2012). The fine-scale architecture of structural variants in 17 mouse genomes. Genome Biology 13(3). doi: 10.1186/gb-2012-13-3-r18

Yang, J. A., Lee, S. H., Goddard, M. E., and Visscher, P. M. (2011). GCTA: a tool for genome-wide complex trait analysis. Am. J. Hum. Gen. 88, 76-82. doi: 10.1016/j.ajhg.2010.11.011

Yang, R. F., Fang, S. Y., Wang, J., Zhang, C. Y., Zhang, R., Liu, D., et al. (2017). Genome-wide analysis of structural variants reveals genetic differences in Chinese pigs. PLoS ONE 12:e186721. doi: 10.1371/journal.pone.0186721

Ye, K., Schulz, M. H., Long, Q., Apweiler, R., and Ning, Z. M. (2009). Pindel: a pattern growth approach to detect break points of large deletions and medium sized insertions from paired-end short reads. Bioinformatics 25, 2865-2871. doi: 10.1093/bioinformatics/btp394

Zhao, P. J., Li, J. H., Kang, H. M., Wang, H. F., Fan, Z. Y., Yin, Z. J., et al. (2016). Structural variant detection by large-scale sequencing reveals new evolutionary evidence on breed divergence between Chinese and European Pigs. Sci. Rep. 6:18501. doi: 10.1038/srep18501

Zhao, P. J., Yu, Y., Feng, W., Du, H., Yu, J., Kang, H. M., et al. (2018). Evidence of evolutionary history and selective sweeps in the genome of Meishan pig reveals its genetic and phenotypic characterization. Gigascience 7:58. doi: 10.1093/gigascience/giy058

Zhou, J., Lemos, B., Dopman, E. B., and Hartl, D. L. (2011). Copy-number variation: the balance between gene dosage and expression in Drosophila melanogaster. Gen. Biol. Evol. 3, 1014-1024. doi: 10.1093/gbe/evr023

Zhou, Z. K., Jiang, Y., Wang, Z., Gou, Z. H., Lyu, J., Li, W. Y., et al. (2015). Resequencing 302 wild and cultivated accessions identifies genes related to domestication and improvement in soybean. Nat. Biotechnol. 33, 408-U125. doi: $10.1038 /$ nbt.3096 
Zhuang, J. L., and Weng, Z. P. (2015). Local sequence assembly reveals a highresolution profile of somatic structural variations in 97 cancer genomes. Nucleic Acids Res. 43, 8146-8156. doi: 10.1093/nar/gkv831

Conflict of Interest: The authors declare that the research was conducted in the absence of any commercial or financial relationships that could be construed as a potential conflict of interest.
Copyright (C) $2021 \mathrm{Du}$, Zheng, Zhao, Hu, Wang, Zhou and Liu. This is an open-access article distributed under the terms of the Creative Commons Attribution License (CC $B Y)$. The use, distribution or reproduction in other forums is permitted, provided the original author(s) and the copyright owner(s) are credited and that the original publication in this journal is cited, in accordance with accepted academic practice. No use, distribution or reproduction is permitted which does not comply with these terms. 